\title{
Standards for Internet-Based Experimenting
}

\author{
Ulf-Dietrich Reips
}

University of Zürich, Switzerland

\begin{abstract}
This article summarizes expertise gleaned from the first years of Internet-based experimental research and presents recommendations on: (1) ideal circumstances for conducting a study on the Internet; (2) what precautions have to be undertaken in Web experimental design; (3) which techniques have proven useful in Web experimenting; (4) which frequent errors and misconceptions need to be avoided; and (5) what should be reported. Procedures and solutions for typical challenges in Web experimenting are discussed. Topics covered include randomization, recruitment of samples, generalizability, dropout, experimental control, identity checks, multiple submissions, configuration errors, control of motivational confounding, and pre-testing. Several techniques are explained, including "warm-up," "high hurdle," password methods, "multiple site entry," randomization, and the use of incentives. The article concludes by proposing sixteen standards for Internet-based experimenting.
\end{abstract}

Key words: Internet-based experimenting, Web experiment, standards, experiment method, psychological experiment, online research, Internet research, Internet science, methodology

\section{Introduction}

We are in the midst of an Internet revolution in experimental research. Beginning in the mid-nineties of the last century, using the world-wide network for experimental research became the method of choice for a small number of pioneers. Their early work was conducted soon after the invention of forms on Web pages established user-server interaction (Musch \& Reips, 2000). This medium holds the promise to achieve further methodological and procedural advantages for the experimental method and a previously unseen ease of data collection for scientists and students.

Several terms are used synonymously for Internet-based experiments: Web experiment, on(-)line experiment, Web-based experiment, World Wide Web $(W W W)$ experiment, and Internet experiment. Here the term Web experiment will be used most often because historically this term was used first and ex-

I would like to thank Tom Buchanan, William C. Schmidt, Jochen Musch, Kevin O’Neil and an anonymous reviewer for very helpful comments on earlier versions of this article. periments delivered via the Web are clearly the most accessible and popular, as experiments using Internet services other than the Web (such as e-mail, ICQ, Telnet, Gopher, FTP, etc.) are rarely conducted. Many of the issues discussed in this article apply to experiments conducted via these services as well, and even to nonexperimental Internet-based methods, such as Web surveying or nonreactive data collection (for examples see Reips \& Bosnjak, 2001).

Web experiments may be used to validate results from field research and from laboratory experiments (see Krantz \& Dalal, 2000; Pohl, Bender, \& Lachmann, 2002, this volume; Reips, 1997; Reips, Morger, \& Meier, 2001), or they may be used for new investigations that could only be feasibly accomplished in this medium. For instance, in 2000, Klauer, Musch, and Naumer published an article on belief bias in Psychological Review that contains an example of a study that reasonably could only be conducted as an Internet-based experiment, because several thousand participants were needed to obtain accurate estimates of model parameters. Similarly, Birnbaum (2001) recruited experts in decision making via a decision making researchers' e-mail list and sent these experts to a Web page where many of them contradicted their own theories in a Web experiment on choices between gambles. Experiments such as 
these would prove impractical and burdensome if delivered in another medium.

In addition to the benefit of increased access to participants, Internet-based experimenting always includes the possibility to use the programmed experimental materials in a traditional laboratory setting (Reips, 1997). In contrast, a laboratory experiment, even if built with Internet software technologies, cannot simply be turned into a Web experiment by connecting it to the Internet. Successful and appropriate use of the Web medium requires careful crafting and demands methodological, procedural, technical, and ethical considerations to be taken into account! While laboratory experiments can be built directly with Internet software technologies, it seems wise to conceptualize experiments as Web experiments whenever possible, given their many advantages.

Sheer numbers, reduced cost, and accessibility of specific participants are only a few of the Internetspecific properties in Web experimenting that create an environment that has been greeted with great enthusiasm by experimental psychologists.

\section{When and When not to Conduct an Experiment on the Internet?}

Before standards for Internet-based experimenting can be established, a few words should be devoted to the question of criteria that should be used in deciding the mode an experiment is best conducted in.

\section{Implementing an Experiment: A General Principle}

Because many laboratory experiments are conducted on computers anyway, nothing is lost when an experiment is designed Web-ready: It can always also be used in the laboratory. In distributed Web experimenting, local collaborators recruit and assist participants who all log onto the same Internet-based experiment (Reips, 1999).

\section{Solving Long-Standing Issues in Experimental Research}

The experimental method has a long and successful tradition in psychological research. Nevertheless, the method has been criticized, particularly in the late 1960s and early 1970s (e.g., Chapanis, 1970; Orne, 1962; Rosenthal, 1966; Rosenthal \& Fode, 1973; Rosenthal \& Rosnow, 1969; Smart, 1966). This criticism is aimed in part at the validity of the method and in part at improper aspects of its realization; for instance experimenter effects, volunteer bias, and low power. A solution for many of these problems could be the implementation of experiments as Web experiments. In total, about eighteen advantages counter seven disadvantages of Web experimenting (Reips, 2000, see Table 1).

\section{Why Experimenters Relish Internet-Based Experimenting}

Speed, low cost, external validity, experimenting around the clock, a high degree of automation of the experiment (low maintenance, limited experimenter effects), and a wider sample are reasons why the Internet may be the setting of choice for an experiment. Seventy percent of those who have conducted a Web experiment intend to certainly use this method again (the other $30 \%$ maybe). This result from a survey of many of the early pioneers in Web experimenting conducted by Musch and Reips (2000) is indirect evidence that learning and using the methods of Internet-based experimenting is certainly worthwhile. Surveyed Web experimenters rated "large number of participants" and "high statistical power" as the two most important factors why they made the decision to conduct a Web experiment.

The survey conducted by Musch and Reips is in itself a good example that Internet-based research may be the method of choice if a special subpopulation is to be reached. Access to specific groups can be achieved through Internet newsgroups (Hewson, Laurent, \& Vogel, 1996; Schmidt, 1997), Web site guestbooks, chat forums, or topic-related mailing lists. Eichstaedt (2002, Experiment 1) recruited persons using either Macintosh or Windows operating systems for his Web experiment via newsgroups devoted to the discussion of issues related to these operating systems. The participants performed a Javabased tachistoscopic word recognition task that included words typically used in ads for these computer systems. Word recognition was faster for words pertaining to a participant's computer system. Some target groups may be easier to study via Internet, because persons belonging to this group will only reveal critical information under the protection of anonymity, for example drug dealers (Coomber, 1997), or Ecstasy users (Rodgers, Buchanan, Scholey, Heffernan, Ling, \& Parrott, 2001).

\section{When Not to Conduct an Experiment on the Internet}

Obviously, Web experiments are not the most suitable method for all research projects. For instance 
Table 1. Web Experiments: Advantages, Disadvantages and Solutions (Adapted from Reips, 2000)

Advantages of Web Experiments

(1) Ease of access to a large number of demographically and culturally diverse participants (for an example of a study conducted in three languages with 440 women from more than nine countries see in this volume Bohner, Danner, Siebler, \& Samson, 2002);

(2) ... as well as to rare and specific participant populations (Schmidt, 1997).

(3) Better generalizability of findings to the general population (Horswill \& Coster, 2001; Reips, 1995).

(4) Generalizability of findings to more settings and situations (because of high external validity, e.g., Laugwitz, 2001).

(5) Avoidance of time constraints.

(6) Avoidance of organizational problems, such as scheduling difficulties, as thousands of participants may participate simultaneously.

(7) Highly voluntary participation.

(8) Ease of acquisition of just the optimal number of participants for achieving high statistical power while being able to draw meaningful conclusions from the experiment.

(9) Detectability of motivational confounding.

(10) Reduction of experimenter effects.

(11) Reduction of demand characteristics.

(12) Cost savings of laboratory space, personnel hours, equipment, administration.

(13) Greater openness of the research process (increases replicability).

(14) Access to the number of nonparticipants.

(15) Ease of comparing results with results from a locally tested sample.

(16) Greater external validity through greater technical variance.

(17) Ease of access for participants (bringing the experiment to the participant instead of the opposite).

(18) Public control of ethical standards.
Disadvantages with Solutions

(1) Possible multiple submissions - can be avoided or controlled by collecting personal identification items, by checking internal consistency as well as date and time consistency of answers (Schmidt, 1997), and by using techniques such as sub-sampling, participant pools, or handing out passwords (Reips, $1999,2000,2002 b)$. There is evidence that multiple submissions are rare in Web experiments (Reips, 1997).

(2) Generally, experimental control may be an issue in some experimental designs, but is less of an issue when using between-subjects designs with random distribution of participants to experimental conditions.

(3) Self-selection can be controlled by using the multiple site entry technique.

(4) Dropout is always an issue in Web experiments. However, dropout can be turned into a detection device for motivational confounding. Also, dropout can be reduced by implementing a number of measures, such as promising immediate feedback, giving financial incentives, and by personalization (Frick, Bächtiger, \& Reips, 2001).

(5) The reduced or absent interaction with participants during a Web experiment creates problems, if instructions are misunderstood. Possible solutions are pretests of the materials and providing the participants with the opportunity for giving feedback.

(6) The comparative basis for the Web experiment method is relatively low. This will change.

(7) External validity of Web experiments may be limited by their dependence on computers and networks. Also, many studies cannot be done on the Web. However, where comparable, results from Web and laboratory studies are often identical (Krantz \& Dalal, 2000). whenever physiological parameters of participants are to be measured directly, specialized hardware is required, or when a tightly controlled setting is important, then laboratory experiment administration is still required.

A further basic limitation lies in Web experiments' dependency on computers and networks hav- ing psychological, technical, and methodological implications. Psychologically, participants at computers will likely be subject to self-actualization and other influences in computer-mediated communication (e.g., Bargh, McKenna, \& Fitzsimons, 2002; Joinson, 2001). Technically, more variance is introduced in the data when collected on the Internet than in the 
laboratory, because of varying network connection speed, varying computer speed, multiple software running in parallel, etc. (Reips, 1997, 2000).

On first view one may think that the Internet allows for easy setup of intercultural studies, and it is certainly possible to reach people born into a wide range of cultures. However, it is a widespread misunderstanding that Internet-based cultural research would somehow render unnecessary the use of many techniques that have been developed by cross-cultural psychologists (such as translation - back translation, use of the studied cultures' languages, and extensive communication and pretests with people from the cultures that are examined). Issues of access, self-selection, and sampling need to be resolved. In many cultures, English-speaking computer users are certainly not representative of the general population. Nevertheless, these people may be very useful in bridging between cultures, for instance, in cooperative studies based on distributed Web experimenting.

Finally, for ethical reasons, many experiments cannot be conducted that require an immediate debriefing and adjunctive procedures through direct contact whenever a participant terminates participation.

In the following section we will turn to central issues and resulting proposals for standards that specifically apply to Internet-based experimenting.

\section{Checks and Solutions for Methodological Challenges in Web Studies}

The following section contains methodological and technical procedures that will reduce or alleviate issues that are rooted within the very nature of Internet-based studies.

\section{Web Experiment Implementation}

Data collection techniques on the Internet can be polarized into server-side and client-side processing. Server-side methods (a Web server, often in combination with a database application, serves Web pages that can be dynamically created depending on a user's input, Schmidt, 2000) are less prone to platformdependent issues, because dynamic procedures are performed on the server so that they are not subject to technical variance. Client-side methods use the processing power of the participants' computers. Therefore, time measurements do not contain error from network traffic and problems with server avail- ability are less likely. Such measurements do rely on the user's computer configuration however (Schmidt, 2000). Combinations of server-side and client-side processing methods are possible; they can be used to estimate technical error variance by comparison of measurements.

General experimental techniques apply to Web experimentation as well. For example, randomized distribution of participants to experimental conditions is a measure against confounding and helps avoiding order effects. Consequently, in every Web experiment at least one randomization technique should be used. In order of reliability, these are roughly: (1) CGI or other server-side solutions, (2) client-side Java, (3) Javascript, and (4) "the birthday technique" (participants pick their experimental conditions by mouse-clicking on their birthday or birthday month; Birnbaum, 2000; Reips, 2002b).

\section{Generating an Experiment}

For several years, Web experimenters created their experimental materials and procedures "by hand." With enough knowledge about HTML and the services and structures available on the Internet conducting a Web experiment was only moderately complicated. However, many researchers hesitate before acquiring new technical skills. Fortunately, several recently developed applications considerably ease the development of a Web experiment. For within-subjects designs, Birnbaum (2000) developed FactorWiz, ${ }^{1}$ a Web page that creates Web pages with items combined according to previously defined factorial designs. WEXTOR, ${ }^{2}$ by Reips and Neuhaus (2002), a Web-based tool for generating and visualizing experimental designs and procedures, guides the user through a ten-step program of designing an experiment that may include between-subjects, withinsubjects, and quasi-experimental (natural) factors. WEXTOR automatically creates the experiments in such a way that certain methodological requirements of Internet-based experimentation are met (for example, nonobvious file naming [Reips, 2002a] is implemented for experimental materials and conditions, and a session ID is generated that helps identify submissions by the same participant).

\section{Recruitment}

A Web experiment can be announced as part of the collection of Web studies by the American Psycho-

\footnotetext{
${ }^{1} \mathrm{http}: / /$ psych.fullerton.edu/mbirnbaum/programs/ factorWiz.htm

${ }^{2}$ http://www.genpsylab.unizh.ch/wextor/index.html
} 
logical Society. ${ }^{3}$ This Web site is maintained by John Krantz. A virtual laboratory for Web experiments is the Web Experimental Psychology Lab ${ }^{4}$. This Web site is visited by about 4500 potential participants a month (Reips, 2001). Internet-based experiments should always be linked to the web experiment list, ${ }^{5}$ a Web site that is intended to serve as an archive of links and descriptions of as many experiments conducted on the Internet as possible. Other ways of recruiting participants that may be combined with linking to experiment Web sites is the use of online panels, newsgroups, search engines, banners, and e-mail lists. Also, participants for Web experiments can be recruited offline (e.g., Bamert, 2002; Eichstaedt, 2002, this volume; Pohl et al., 2002; Reips et al., 2001; Ruppertsberg, Givaty, Van Veen, \& Bülthoff, 2001).

\section{Generalizability}

\section{Self-Selection}

In many areas of Psychology self-selection is not considered much of a problem in research because theory testing is the underlying model of epistemology and people are not considered to vary much on the essential criteria, for example, in research on cognition and perception. However, at least in research more socially oriented, self-selection may interfere with the aim of the study at hand and limit its generalizability. The presence and impact of self-selection in an Internet-based study can be tested by using the multiple site entry technique (Reips, 2000, 2002b). Via log file analysis it is possible to determine a Web experiment's degree of appeal for participation for each of the samples associated with referring Web sites.

The multiple site entry technique can be used in any Internet-based study (for a recent example of longitudinal trauma survey research implementing this technique see Hiskey \& Troop, 2002). Several links to the study are placed on Web sites, in discussion groups, or other Internet forums that are likely to attract different types of participants. Placing identifying information in the published URLs and analyzing different referrer information in the HTTP protocol can be used to identify these sources (see Schmidt, 2000). Later the data sets that were collected are compared for differences in relative degree of appeal (measured via dropout), demographic data, central results, and data quality, as a function of re-

\footnotetext{
3 http://psych.hanover.edu/APS/exponnet.html

$4 \mathrm{http}$ ://www.genpsy.unizh.ch/Ulf/Lab/ WebExpPsyLab.html

5 http://www.genpsy.unizh.ch/Ulf/Lab/webexplist.html
}

ferring location. Consequently, an estimate of biasing potential through self-selection can be calculated (Reips, 2000). If self-selection is not found to be a problem, results from psychological experiments should be the same no matter where participants are recruited, and should therefore show high generalizability.

\section{Dependence on Technology}

Limited generalizability of results from Internetbased research may also arise due to dependence on computers and networking technology. Web-based experimenting can be seen as a form of computermediated communication. Hence, differences found in comparisons between behaviors in computer-mediated situations and face-to-face situations (see, for example, Buchanan, 2002; Kiesler \& Sproull, 1986; Postmes, Spears, Sakhel, \& DeGroot, 2001) need to be taken into account when results from online research are interpreted.

\section{Advantages}

Apart from the challenges to generalizability mentioned above, Internet-based experimenting has three major advantages in this respect:

(1) Increased generalizability through nonlocal samples with a wider distribution of demographic characteristics (for a comparison of data from several Web experiments see Krantz \& Dalal, 2000, and Reips, 2001).

(2) "Ecological" validity: "the experiment comes to the participant, not vice versa" (Reips, 1995, 1997). Participants in Web experiments often remain in familiar settings (e.g., at their computer at home or at work) while they take part in an Internet-based experiment. Thus, any effects cannot be attributed to being in an unfamiliar setting.

(3) The high degree of voluntariness - because there are fewer constraints on the decisions to participate and to continue participation, the behaviors observed in Internet-based experiments may be more authentic and therefore can be generalized to a larger set of situations (Reips, 1997, 2000).

A high degree of voluntariness is a trade-off for potential effects of self-selection. Voluntariness refers to the voluntary motivational nature of a person's participation, during the initial decision to participate and during the course of the experiment session. It is influenced by external factors, for example, the setting, the experimenter, and institutional regulations. If participants in an experiment subjectively 
feel that they are participating entirely voluntarily as opposed to feeling somewhat coerced into participating (as is often the case in the physical laboratory), then they are more likely to initiate and complete an experiment, and to comply with the instructions.

The participant's impression of voluntariness reduces effects of psychological reactance (actions following an unpleasant feeling brought about by a perceived threat to one's freedom - such as being "made" to do something by an experimenter); effects such as careless responding, deliberately false answers, and ceasing participation. Therefore, the dropout rate in Web experiments can serve as an indicator of the reactance potential of an experiment. Generally, participant voluntariness is larger in Web experiments than in laboratory experiments (Reips, 1997, 2000).

Although Web experiments can introduce a reliance on technology, their improved "ecological" validity may be seen as a counterweight to this factor when contemplating arguments in favor and opposition of one's choice of method.

\section{Dropout: Avoiding it, Reducing its Impact, Using it}

Dropout curves (dropout progress), or at least dropout rates (attrition rates, or the opposite: return rates) should be reported for all studies conducted on the Internet, separately for all between-subjects experimental conditions. Dropout may pose similar problems as self-selection. However, dropout concerns the end of participation (a comprehensive discussion of other forms of nonresponse is found in Bosnjak, 2001) instead of a decision to initiate participation. If participants end their participation selectively, then the explanatory power of the experiment is severely compromised, especially if dropout varies systematically with levels of the independent variable(s) or with certain combinations of levels. For example, if the impact of two different tasks is to be measured, one of which is more boring, then participants are more likely to drop from that condition. In this case motivational confounding is present (Reips, 2000, 2002a, 2002b).

Two types of evasive measures can be taken to counter the problem of dropout: (1) reducing dropout, and (2) reducing the negative impact of dropout.

\section{Measures to be Taken in Reducing Dropout}

Dropout is reduced by all measures that form a motivating counterweight to the factors promoting dropout. For instance, some dropout reducing measures include the announcement of chances for financial incentives and questions about personal information at the beginning of the Web experiment (Frick et al., 2001; Musch \& Reips, 2000; but see O'Neil \& Penrod, 2001). It is potentially biasing to use scripts that do not allow participants to leave any items unanswered. If the average time needed to fill in an experiment questionnaire is longer than a few minutes, then data quality may suffer from psychological reactance or even anger by those participants who do not wish to answer all questions, because some participants will fill in answers randomly and others will drop out.

If all or most of the items in a study are placed on a single Web page then meaningful dropout rates can not be reported: we do not know at which item people decide to drop out. In the terminology of Bosnjak (2001), such a design is not suited to distinguish unit nonresponders from item nonresponders, item nonresponding dropouts, answering dropouts, lurking dropouts, lurkers, and complete responders. They all appear as either dropouts or complete responders, and it becomes impossible to measure a decision not to participate separately from a decision to terminate participation. Consequently, a "oneitem-one-screen" design or at least a multipage design (with a parcel of items on each page) is recommended. Layout, design, loading time, appeal, and functionality of the experimental materials play a role as well (Reips, 2000, 2002b; Wenzel, 2001).

Sophisticated technologies and software used in Internet research (e.g., Javascript, Flash, multimedia elements such as various audio or streaming video formats as part of Web experiments, or experiments written in Authorware or as Java applets) may contribute to dropout (Schmidt, 2000). Potential participants unable or unwilling to run such software will be excluded from the start, and others will be dropped, if resident software on their computer interacts negatively with these technologies (Schwarz \& Reips, 2001). Findings by Buchanan and Reips (2001) indicate that samples obtained using such technologies will inevitably incorporate biases: People using certain technologies differ psychologically from those who don't. Buchanan and Reips showed that there are personality differences between different groups of respondents: Mac users scored higher on Openness, and people in the Javascriptenabled condition had lower average education levels than those who could or did not have Javascript enabled in their browsers. Consequently, whenever possible, Internet studies should be conducted using only the most basic and widely available technology.

\section{Measures to be Taken in Reducing the Negative Impact of Dropout}

Sometimes, there might be no alternative to avoiding the negative impact of dropout by forgoing self-se- 
Table 2. Checklist High-Hurdle Technique

\begin{tabular}{ll}
\hline Component & Description \\
\hline $\begin{array}{l}\text { Seriousness } \\
\text { Personalization }\end{array}$ & $\begin{array}{l}\text { Tell participants participation is serious, and that science needs good data. } \\
\text { Ask for e-mail address and/or phone number, and other personal data. }\end{array}$ \\
Impression of control & $\begin{array}{l}\text { Tell participants that their identity can be traced (for instance, via their comput- } \\
\text { er's IP address). }\end{array}$ \\
Patience: Loading time & $\begin{array}{l}\text { Use image files to successively reduce the loading time of Web pages. } \\
\text { Place most text on the first page of the Web experiment, and reduce amount } \\
\text { page by page. }\end{array}$ \\
Patience: Long texts & $\begin{array}{l}\text { Give an estimate of how long participation in the Web experiment will take. } \\
\text { Prepare the participants for any sensitive aspects of your experiment (e.g., "you } \\
\text { Privacy }\end{array}$ \\
Preconditions & $\begin{array}{l}\text { Name asked about your financial situation"). } \\
\text { Perform tests for compatibility of Java, JavaScript, and other technologies, if ap- }\end{array}$ \\
pechnical pretests & $\begin{array}{l}\text { plicable. } \\
\text { Indicate extra rewards for full compliance. }\end{array}$ \\
\hline
\end{tabular}

lected Internet participation and choosing to conduct the study with a precommitted offline sample. If the absence of dropout is crucial, a dropout-oriented experimental design may help. Three simple techniques can be used to reduce dropout: high hurdle, seriousness check, and warm-up. All three of these techniques have been repeatedly used in the Web Experimental Psychology Lab (e.g., Musch \& Klauer, 2002; Reips, 2000, 2001; Reips et al., 2001).

In the high-hurdle technique, motivationally adverse factors are announced or concentrated as close to the beginning of the Web experiment as possible. On the following pages, the concentration and impact of these factors should be reduced continuously. As a result, the highest likelihood for dropout resulting from these factors will be at the beginning of the Web experiment. The checklist in Table 2 shows factors to be bundled and measures to be taken in the high-hurdle technique (also see Reips, 2000).

A second precaution that can be taken to reduce dropout is asking for the degree of seriousness of a participant's involvement (Musch \& Klauer, 2002) or for a probability estimate that one will complete the whole experiment. If it is pre-determined that only data sets from persons with a motivation for serious participation will be analyzed, then the resulting dropout rate is usually much lower.

The warm-up technique is based on the observation that most dropout will take place at the beginning of an online study, forming a "natural dropout curve" (Reips, 2002b). A main reason for the initial dropout is the short orientation period many participants show before making a final decision on their participation (orientation often takes place even after clicking on a submit button confirming "informed consent," because informed consent forms tend to be filled with abstract "legalese" while the study materi- als are concrete ${ }^{6}$ ). To keep dropout low during the experimental phase, as defined by the occurrence of the experimental manipulation, it is wise to place its beginning several Web pages deep into the study. The warm-up period can be used for practice trials, piloting of similar materials or buildup of behavioral routines, and assurance that participants are complying with instructions. Figure 1 shows the resulting dropout during warm-up phase and experimental phase after implementation of the warm-up technique in the experiment by Reips et al. (2001).

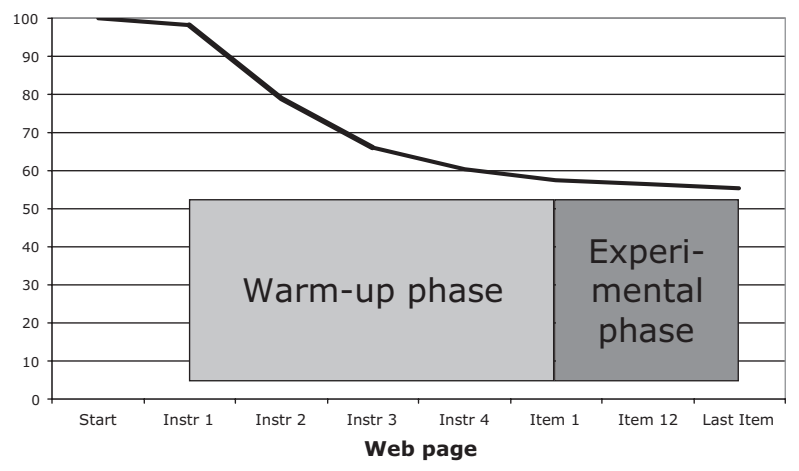

Figure 1. Percentage of remaining participants as a function of progress into the Web experiment by Reips et al. (2001). The implementation of the warmup technique resulted in very low dropout after introduction of the experimental manipulation (beginning with "Item 1").

6 At Central European research institutions, informed consent is often provided in the form of a brief statement that the person will become a participant in a study by going further, accompanied by some (again: brief) information about the research institution, the study and the researchers. Many researchers feel that participants are per 
Dropout may also be used as a dependent variable (Frick et al., 2001; Reips, 2002a), for example in usability research.

\section{Control}

\section{Experimental Setting}

It can be seen as a major problem in Web experimenting that the experimenter has limited control of the experimental setting 7 . For example, in Web-based perception experiments it is more difficult than in the laboratory to guarantee that stimuli are perceived as intended or even assess how they were presented (Krantz, 2000, 2001). While we cannot control this principal property of Web experimentation, we can certainly use technical measures to collect information about the computer-bound aspects of the setting at the participant's end of the line. Via HTTP protocol, Javascript, and Java, the following information can be accessed: (1) type and version of Web browser, (2) type and version of operating system, (3) screen width and height, (4) screen resolution, (5) color depth of monitor setting, (6) accuracy of the computer's timing response (Eichstaedt, 2001), and (7) loading times.

Javascript, Java, and plug-in based procedures may inherently reduce experimental control, because these programming languages and routines often interact with other software on the client computer. They will lead to an increase in loading times of Web pages, and increase the likelihood of inaccessibility of the Web experiment. A study by Schwarz and Reips (2001) showed that an otherwise identical copy of a Web experiment had a $13 \%$ higher dropout rate if Javascript was used to implement client-side routines for certain experimental procedures. Therefore, the cons of using Javascript and Java carefully need to be considered against the value of gathering the above-listed information. These methods can, however, produce timing capabilities unavailable to any other methods.

\footnotetext{
se knowledgeable about their basic rights and need not be coerced into reading pages of legalese texts. This seems particularly true in Internet-based studies, where the end of participation is always only one mouse click away. Also, lengthy "informed consent forms" may have a biasing impact on the resulting data.

7 I prefer the view that reduced control of situational variables allows for wider generalizability of results, if an effect is found.
}

\section{Identity}

Multiple submissions and reduced quality of data (missing responses, etc.) create potential problems in Internet-based research, because a participant's identity can hardly be determined without doubt. Several scenarios are thinkable:

- The same person uses the same computer (IP address) to participate repeatedly.

- The same person uses different computers to participate repeatedly.

- Different persons use the same computer to participate.

- Multiple datasets are submitted from the same computer, but are assigned different IP addresses.

- Multiple datasets are submitted from different computers, but some Web pages or page elements are assigned the same IP addresses by proxy servers.

There are many indications that the rate of repeated participations (below 3\% in most studies) is not a threat to the reliability of Internet-based research (Krantz \& Dalal, 2000; Musch \& Reips, 2000; Reips, 1997). However, adjunctive measures should be taken and be reported. Data quality is better in Webbased studies if identifying information is asked at the study's onset (Frick et al. 2001). Many attempts at reducing the number of multiple submissions and increasing data quality concentrate on uncovering the identities of participants or computers. Also, in light of the high numbers of participants that can be recruited on the Internet certain data sets can easily be excluded following clear predetermined criteria.

In order to solve the identity problem and resulting issues with data quality the techniques listed in Table 3 may be used in Web experimenting. These techniques can be grouped as techniques of avoidance and techniques of control of multiple submissions.

Users will return to a Web experiment for repeated participation only when they are motivated by entertainment or other attractive features of the experiment. Multiple submissions are likely in Web experiments that are designed in game style (e.g., Ruppertsberg et al., 2001), with highly surprising or entertaining outcomes (for example, the "magic" experiment in the Web Experimental Psychology Lab, available at http://www.genpsylab.unizh.ch/88497/ magic.htm) or with high incentives.

\section{Quality of Data}

Some of the techniques that were discussed earlier in this article are suited to maximize or improve data quality in Web experimentation. For example, Frick 
Table 3. Avoidance and Control of Multiple Submissions

Avoidance of Multiple Submissions Control of Multiple Submissions

(1) Informing participants on the experiment's start page that multiple submissions are unwanted and detrimental to the study's purpose.

(2) Redirecting the second and all further requests for the experiment's start page coming from the same IP address (this technique likely excludes a number of people using Internet providers with dynamic IP addressing).

(3) Implementation of password-dependent access. Every participant is given a personal password that can only be used once (e.g., Schmidt, 2000). Of course, the password scheme should be not too obvious (e.g., do not use "hgz3," "hgz4," "hgz5," etc. as passwords).

(4) Limiting participation to members of a controlled group (participant pool or online panel).

(1) Collecting information that allows personal identification.

(2) Asking participants on the experiment's start page whether they have participated before.

(3) Continuous session IDs created through dynamic hidden variables or Javascript, in combination with analysis of HTTP information (e.g., browser type and operating system).

(4) Use of the sub-sampling technique (Reips, 2000, 2002b): for a limited random sample from all data sets every measure is taken to verify the participants' verifiable responses (for example, age, sex, occupation) and identity, resulting in an estimate for the total percentage of wrong answers and multiple submissions.

(5) Limiting participation to members of a controlled group.

(6) Controlling for internal consistency of answers.

(7) Controlling for consistency of response times.

(8) Placement of identifiers (cookies) on the hard disks of participants' computers - however, this technique is of limited reliability and will exclude a large portion of participants.

et al. (2001) found that participants who requested the Web page with questions about their age, gender, nationality, and e-mail address substantially more often completed responses when these personal data were collected at the beginning $(95.8 \%)$ rather than at the end of an experiment $(88.2 \%)$. The warm-up technique ensures that data collected in the experimental phase come from the mostly highly committed participants. Providing information about incentives for participation certainly helps, but may not be legal in some jurisdictions (for example, in Germany) if the incentive is in the form of sweepstakes and entry is dependent on complete participation. Missing responses can be reduced by making participants aware that they might have accidentally skipped the corresponding items or even by repeatedly presenting these items. Consistency checks help in detecting inappropriate or possibly erroneous answers, for example participants claiming to be 15 years old and having a Ph.D. Quality of data may also be ensured by excluding data sets that do not meet certain criteria (e.g., more than $\times$ missing entries, overly long response times).

In general, incomplete data sets are more frequent in Internet-based research than in offline research (unless the experimenter forces participants into a decision between complete responding or dropout). Fortunately, there is evidence that complete data sets collected online are mostly accurate: Voracek, Stieger, and Gindl (2001) were able to control responses for biological sex via university records in an online study conducted at the University of Vienna. In contradiction to the frequently heard "online gender swapping" myth, they found that the rate of seemingly false responses was below $3 \%$. Furthermore, the data pattern of the participants suspected of false responding was in accordance with their reported biological sex, leading to the conclusion that many of these cases were opposite-sex persons using a friend's or roommate's university e-mail account.

\section{Misunderstandings and Limited Ways of Interaction}

In laboratory experiments it is often necessary to explain some of the procedures and materials to the participants in an interactive communication. In the laboratory, experimenters are able to answer questions directly and verify through dialogue and read- 
ing of nonverbal behavior that the instructions were understood. In Web experiments such interaction is difficult to achieve, and mostly not desired, because it would counter some of the method's advantages, for instance its low expenditure and the ability to collect data around the clock.

Pretesting of the materials and collecting feedback by providing communication opportunities for participants are the most practical ways of avoiding misunderstandings. They should be used with care, in order to identify problems early on.

\section{Errors and Frequent Misconceptions in Internet- Based Experimenting}

Apart from preventing misunderstandings on part of participants careful pretesting and monitoring of an Internet-based experiment will help in detecting the presence of any of a number of frequently made configuration errors and possibly of misconceptions on part of the experimenter.

\section{Configuration Errors and Technical Limitations}

\section{Configuration Errors}

There are five frequently observed configuration errors in Internet-based experimenting (Reips, 2002a) that can easily be avoided, if awareness of their presence is raised and precautions are taken. These errors are:

- Allowing external access to unprotected directories (configuration error I). In most cases, placing a file named "index.htm" or "index.html" into each directory will solve the problem.

- Public display of confidential participant data through URL - data may be written to a third party Web server (configuration error II). To avoid this error, do not collect data with the GET method $^{8}$ on any Web page that is two nodes away from a Web page with links to external sources.

- File and folder names and/or field names reveal the experiment's structure (configuration error

8 The GET method is a request method used in the WWW transmission protocol. The two most often used methods for transmitting form data are GET and POST. Information from a form using the GET method is appended onto the end of the action address being requested; for example, in http://www.genpsylab.unizh.ch?webexp= yes, the answer "yes" in an item "webexp" was appended to the URL of the Web page that a user's action (pressing a submit button, etc.) leads to.
III). Use a mixture of logical and random letters and numbers to avoid this error.

- Ignorance towards the technical variance present in the Internet (configuration error IV). Differences in Web browsers, versions of Web browsers, net connections, hardware components etc. need to be considered in the implementation.

- Biased results from improper use of form elements. For example, if no neutral answer (e.g., "Choose here") is preselected (configuration error V), there may be errors of omission. For example, one might erroneously believe that many seniors participated in an experiment until one realizes that the preset answer in the pull-down menu for age was "69 or older"... Biasing may also result from order effects or type of form element used (i.e., pull-down menus, select multiples in lists, etc.).

\section{Technical Limitations}

There are several technical and methodological sources of error and bias that need to be considered. Security holes in operating systems and Web server applications (see Reips, 2002a; Securityfocus.com, n.d.) may allow viruses to shut down the experiment or corrupt data or even let intruders download confidential participant information. While there are significant differences in vulnerability of operating systems, as monitored permanently by Securityfocus.com, any operating system with a Web server collecting data from human participants over the Internet needs to be maintained with the highest degree of responsibility. Another issue of a researcher's responsibility is a frequently observed breach of the basic principle of record taking in research, if only aggregated data are collected. Raw logfiles with full information need to be stored for reanalysis by other researchers and for meta-analyses.

Records on incomplete data sets (dropout figures) should be collected as well! As mentioned, sometimes dynamic IP addressing makes it more difficult to identify coherent data sets. In any event, drawing conclusions about real behavior from Internet data is sometimes difficult, because log data may be ambiguous. For instance, long response times can be caused by the participant's behavior and also by a slow computer or a slow network connection. High dropout rates caused by technical problems (e.g., when using Javascript, Schwarz \& Reips, 2001) may in some cases bias results. As mentioned, personality types correlate with the use of certain technologies (Buchanan \& Reips, 2001), leading to biases in cases of systematic technical incompatibilities.

Finally, due to the public accessibility of many Web experiments, researchers need to be aware of 
one more likely source of bias: participation of experts (colleagues). In an Internet-based experiment on a causal learning phenomenon in cognition (Reips, 1997) $15 \%$ of participants indicated on an "insider" control item that they were working in or studying cognitive psychology. Providing participants with such an item that allows them to look at the experiment without having to fear potential corruption of a colleague's data avoids this bias.

\section{Misconceptions}

There are two misconceptions about Internet-based experimenting that carry dangerous implications that might lead to blind eyes against errors.

\section{Misconception 1: Experimenting on the Web is Just Like Experimenting in the Lab}

The Internet is not a laboratory. Internet participants may end their commitment at any time that they no longer have interest. Even though lab participants can terminate participation at any time as well, they are less likely to given that they are required to face another human and encounter a potentially embarrassing situation when doing so (e.g., Bamert, 2002; Reips, 1997, 2000).

Web experiments are usually online around the clock, and usually they reach a much larger number of people than laboratory experiments. Therefore, overlooking even small factors may have wide consequences. Web experiments are dependent on the quality of networks and are subject to great variance in local settings of participants. Last but not least, experimenting on the Web is much more public than working in the laboratory. In addition to a wider public, one's colleagues will be able to inform themselves in a much more direct way about one's work.

\section{Misconception 2: Experimenting on the Web is Completely Different from Experimenting in the Lab}

Even though Web experiments are always dependent on networks and computers, most laboratory experiments are conducted on computers.

Often a standardized user interface is used to display the experimental materials on the screen. Web browsers are highly standardized user interfaces. Experimental materials made with the help of tools like WEXTOR (Reips \& Neuhaus, 2002) can be used in both laboratory and Internet-based experiments. Creating the materials is quite easy with basic knowl- edge in experimental design and handling of HTML. Fundamental ideas and methodological procedures are the same in Web and physical lab, and similar results have been produced in studies conducted in both settings (for a summary see Krantz and Dalal, 2000).

As mentioned earlier, the combination of laboratory and Internet-based experimenting in distributed Web experimenting shows that Web and lab can be integrated in creative ways to arrive at new variations of the experimental method.

\section{Dynamics of Data Collection}

Internet-based experimenting creates its own dynamics. Once an experiment is online and linked to a variety of Web sites it will be present on the Internet for a long time, even if the site itself is removed (which it shouldn't be - it should be replaced by information about the experiment instead). Some search engines will cache the experiment's contents, and so do some proxy servers, even if anticaching meta tags are used in the Web pages.

Internet-based laboratories often have large numbers of visitors and are linked extensively (Reips, 2001). As a consequence, they may even create pressure to offer new Web experiments within short periods of time to satisfy "the crowd's desires." Meeting these desires creates the risk of producing superfluous data - an issue that is in need of being discussed by the community of Internet scientists. The flood of data bears the danger of losing the sense for best care and attention towards data and participants. Would this loss of reasonable diligence be a simple "more is worth less" phenomenon that could result in long-term attitude changes in researchers? Or would it reflect an interaction of the realm of possibilities of techno-media power with limited education in Internet-based experimenting? In any case, the scientific process in psychology is changing profoundly.

\section{Summary: Sixteen Standards for Internet-Based Experimenting}

In this article, a number of important issues in Internet-based experimenting were discussed. As a consequence, several routines and standards for Internet-based experimenting were proposed.

When reporting Web experiments, the implementation of and specifics about the mentioned techniques should be included. The following list of recommendations summarizes most of what needs to be remembered and may be used as a standards check- 
list when conducting an Internet-based experiment and reporting its results.

Standard 1: Consider using a Web-based software tool to create your experimental materials. Such tools automatically implement standard procedures for Web experiments that can guard against many problems. Examples are WEXTOR and FactorWiz (see footnotes 1 and 2 for URLs). If you use FactorWiz, make sure to protect your participants' data by changing the default procedure of storing them in a publicly accessible data file and be aware that FactorWiz creates only one-page Web experiments, so you will not be able to measure dropout in a meaningful way.

Standard 2: Pretest your experiment for clarity of instructions and availability on different platforms.

Standard 3: Make a decision whether the advantages of non-HTML scripting languages and plug-ins outweigh their disadvantages.

Standard 4: Check your Web experiment for configuration errors (I-V; Reips, 2002a).

Standard 5: Consider linking your Web experiment to several Internet sites and services (multiple site entry technique) to determine effects of self-selection and estimate generalizability.

Standard 6: Run your experiment both online and offline, for comparison.

Standard 7: If dropout is to be avoided, use the warm-up technique.

Standard 8: Use dropout to determine whether there is motivational confounding.

Standard 9: Use the high-hurdle technique, incentive information, and requests for personal information to influence time and degree of dropout.

Standard 10: Ask filter questions (seriousness of participation, expert status, language skills, etc.) at the beginning of the experiment to encourage serious and complete responses.

Standard 11: Check for obvious naming of files, conditions, and, if applicable, passwords.

Standard 12: Consider avoiding multiple submissions by exclusively using participant pools and password techniques.

Standard 13: Perform consistency checks.

Standard 14: Keep experiment $\log$ and other data files for later analyses by members from the scientific community.

Standard 15: Report and analyze dropout curves or at least dropout rates for experimental conditions separately for between-subjects factors.
Standard 16: The experimental materials should be kept available on the Internet, as they will often give a much better impression of what was done than any verbal description could convey.

\section{Conclusion}

Internet-based experimenting is fast becoming a standard method and therefore it is a method that needs standards. Many established experimentalists as well as students are currently making their first attempts in using the new method. So far, in many universities there is no curriculum that teaches Internet-based experimenting. Still only few people have both the technical and the methodological experience to give advice to those who would like to commence with the venture of conducting Web experiments. Without established standards the likelihood is high for making grave errors that would result in loss or reduced quality of data, in biased results, or in breach of ethical practices. Consequently, in the present paper an attempt was made to collect and discuss what has been learned in Internet-based experimenting in order to make recommendations, warn about errors, and introduce useful techniques. As a result, a set of standards for Internet-based experimenting could be defined that hopefully will serve as a guide for future experiments on the Internet.

Those who have done Web experiments keep conducting them. Many of those who haven't will do soon. And many of those who would never conduct an experiment on the Internet will be confronted with the methodology as reviewers and readers of publications or as teachers of students who ask for convenient and proper ways of collecting experimental data in that international communication network that allow us to investigate the psychology of the many distant human beings out there. Hopefully, with the guidance of standards and examples from the present special issue of Experimental Psychology, Internet-based experimenting will come one step closer to being established and used as an equivalent tool for scientists.

\section{References}

Bamert, T. (2002). Integration von Wahrscheinlichkeiten: Verarbeitung von zwei Wahrscheinlichkeitsinformationen [Integration of probabilities: Processing two pieces of probability information]. Unpublished master's thesis, University of Zurich, Switzerland.

Bargh, J. A., McKenna, K. Y. A., \& Fitzsimons, G. M. (2002). Can you see the real me? Activation and expression of the "true self" on the Internet. Journal of Social Issues, 58, 33-48. 
Birnbaum, M. H. (2000). SurveyWiz and FactorWiz: JavaScript Web pages that make HTML forms for research on the Internet. Behavior Research Methods, Instruments, and Computers, 32, 339-346.

Birnbaum, M. H. (2001). A Web-based program of research on decision making. In U.-D. Reips \& M. Bosnjak (Eds.), Dimensions of Internet Science (pp. 2355). Lengerich, Germany: Pabst Science.

Bohner, G., Danner, U. N., Siebler, F., \& Samson, G. B. (2002). Rape myth acceptance and judgments of vulnerability to sexual assault: An Internet experiment. Experimental Psychology, 49 (4), 257-269.

Bosnjak, M. (2001). Participation in non-restricted Web surveys: A typology and explanatory model for item non-response. In U.-D. Reips \& M. Bosnjak (Eds.), Dimensions of Internet Science (pp. 193-208). Lengerich, Germany: Pabst Science.

Buchanan, T. (2002). Online assessment: Desirable or dangerous? Professional Psychology: Research and Practice, 33, 148-154.

Buchanan, T., \& Reips, U.-D. (2001, October 10). Platform-dependent biases in Online Research: Do Mac users really think different? In K. J. Jonas, P. Breuer, B. Schauenburg, \& M. Boos (Eds.), Perspectives on Internet Research: Concepts and Methods. Retrieved December 27, 2001, from http://server3.unipsych.gwdg.de/gor/contrib/buchanan-tom

Chapanis, A. (1970). The relevance of laboratory studies to practical situations. In D. P. Schultz (Ed.), The science of psychology: Critical reflections. New York: Appleton Century Crofts.

Coomber, R. (1997, June 30). Using the Internet for survey research. Sociological Research Online, 2, Retrieved June $16^{\text {th }}, 2002$, from http://www.socresonline.org.uk/ 2/2/2.html

Eichstaedt, J. (2001). Reaction time measurement by JAVA-applets implementing Internet-based experiments. Behavior Research Methods, Instruments, and Computers, 33, 179-186.

Eichstaedt, J. (2002). Measuring differences in preactivation on the Internet: The content category superiority effect. Experimental Psychology, 49 (4), 283-291.

Frick, A., Bächtiger, M. T., \& Reips, U.-D. (2001). Financial incentives, personal information, and dropout in online studies. In U.-D. Reips \& M. Bosnjak (Eds.), Dimensions of Internet Science (pp. 209-219). Lengerich, Germany: Pabst Science.

Hewson, M., Laurent, D., \& Vogel, C. M. (1996). Proper methodologies for psychological and sociological studies conducted via the Internet. Behavior Research Methods, Instruments, and Computers, 28, 186-191.

Hiskey, S., \& Troop, N. A. (2002). Online longitudinal survey research: Viability and participation. Social Science Computer Review, 20 (3), 250-259.

Horswill, M. S., \& Coster, M. E. (2001). User-controlled photographic animations, photograph-based questions, and questionnaires: Three instruments for measuring drivers' risk-taking behavior on the Internet. Behavior Research Methods, Instruments, and Computers, 33, $46-58$.

Joinson, A. (2001). Self-disclosure in computer-mediated communication: The role of self-awareness and visual anonymity. European Journal of Social Psychology, 31, $177-192$.

Kiesler, S., \& Sproull, L. S. (1986). Response effects in the electronic survey. Public Opinion Quarterly, 50, $402-413$.
Klauer, K. C., Musch, J., \& Naumer, B. (2000). On belief bias in syllogistic reasoning. Psychological Review, 107, 852-884.

Krantz, J. H. (2000). Tell me, what did you see? The stimulus on computers. Behavior Research Methods, Instruments, and Computers, 32, 221-229.

Krantz, J. H. (2001). Stimulus delivery on the Web: What can be presented when calibration isn't possible. In U.-D. Reips \& M. Bosnjak (Eds.), Dimensions of Internet Science (pp. 113-130). Lengerich, Germany: Pabst Science.

Krantz, J. H., \& Dalal, R. S. (2000). Validity of Web-based psychological research. In M. H. Birnbaum (Ed.), Psychological experiments on the Internet (pp.35-60). San Diego, CA: Academic Press.

Laugwitz, B. (2001). A Web experiment on color harmony principles applied to computer user interface design. In U.-D. Reips \& M. Bosnjak (Eds.), Dimensions of Internet Science (pp. 131-145). Lengerich, Germany: Pabst Science.

Musch, J., \& Klauer, K. C. (2002). Psychological experimenting on the World Wide Web: Investigating content effects in syllogistic reasoning. In B. Batinic, U.-D. Reips, \& M. Bosnjak (Eds.), Online Social Sciences (pp. 181-212). Göttingen, Germany: Hogrefe.

Musch, J., \& Reips, U.-D. (2000). A brief history of Web experimenting. In M. H. Birnbaum (Ed.), Psychological experiments on the Internet (pp.61-88). San Diego, CA: Academic Press.

O’Neil, K. M., \& Penrod, S. D. (2001). Methodological variables in Web-based research that may affect results: Sample type, monetary incentives, and personal information. Behavior Research Methods, Instruments, and Computers, 33, 226-233.

Orne, M. T. (1962). On the social psychology of the psychological experiment: With particular reference to demand characteristics and their implications. American Psychologist, 17, 776-783.

Pohl, R. F., Bender, M., \& Lachmann, G. (2002). Hindsight bias around the world. Experimental Psychology, 49 (4), 270-282.

Postmes, T., Spears, R., Sakhel, K., \& DeGroot, D. (2001). Social influence in computer-mediated communication: The effect of anonymity on group behavior. Personality and Social Psychology Bulletin, 27, 1243-1254.

Reips, U.-D. (1995). The Web experiment method. Retrieved January 6, 2002, from http://www.genpsy.unizh.ch/Ulf/Lab/WWWExpMethod.html

Reips, U.-D. (1997). Das psychologische Experimentieren im Internet [Psychological experimenting on the Internet]. In B. Batinic (Ed.), Internet für Psychologen (pp. 245-265). Göttingen, Germany: Hogrefe.

Reips, U.-D. (1999). Online research with children. In U.D. Reips, B. Batinic, W. Bandilla, M. Bosnjak, L. Gräf, K. Moser, \& A. Werner (Eds.). Current Internet science - trends, techniques, results. Aktuelle OnlineForschung - Trends, Techniken, Ergebnisse. Zürich: Online Press. Retrieved April 7, 2002 from http:// dgof.de/tband99/

Reips, U.-D. (2000). The Web experiment method: Advantages, disadvantages, and solutions. In M. H. Birnbaum (Ed.), Psychological experiments on the Internet (pp. 89-114). San Diego, CA: Academic Press.

Reips, U.-D. (2001). The Web Experimental Psychology Lab: Five years of data collection on the Internet. $\mathrm{Be}$ havior Research Methods, Instruments, and Computers, 33, 201-211. 
Reips, U.-D. (2002a). Internet-based psychological experimenting: Five dos and five don'ts. Social Science Computer Review, 20 (3), 241-249.

Reips, U.-D. (2002b). Theory and techniques of conducting Web experiments. In B. Batinic, U.-D. Reips, \& M. Bosnjak (Eds.), Online Social Sciences (pp. 229-250). Seattle: Hogrefe \& Huber.

Reips, U.-D., \& Bosnjak, M. (2001). Dimensions of Internet Science. Lengerich, Germany: Pabst Science.

Reips, U.-D., Morger, V., \& Meier B. (2001). "Fünfe gerade sein lassen": Listenkontexteffekte beim Kategorisieren ["Letting five be equal": List context effects in categorization]. Unpublished manuscript. Retrieved April 7, 2002 from http://www.psychologie.unizh.ch/ genpsy/reips/papers/re_mo_me2001.pdf

Reips, U.-D., \& Neuhaus, C. (2002). WEXTOR: A Webbased tool for generating and visualizing experimental designs and procedures. Behavior Research Methods, Instruments, and Computers, 34, 234-240.

Rodgers, J., Buchanan, T., Scholey, A. B., Heffernan, T. M., Ling, J., \& Parrott, A. (2001). Differential effects of Ecstasy and cannabis on self-reports of memory ability: A web-based study. Human Psychopharmacology: Clinical and Experimental, 16, 619-625.

Rosenthal, R. (1966). Experimenter effects in behavioral research. New York: Appleton-Century-Crofts.

Rosenthal, R., \& Fode, K. L. (1973). The effect of experimenter bias on the performance of the albino rat. Behavioral Science, 8, 183-189.

Rosenthal, R., \& Rosnow, R. L. (1969). Artifact in behavioral research. New York: Academic Press.

Ruppertsberg, A. I., Givaty, G., Van Veen, H. A. H. C., \& Bülthoff, H. (2001). Games as research tools for visual perception over the Internet. In U.-D. Reips \& M. Bosnjak (Eds.), Dimensions of Internet Science (pp. 147-158). Lengerich, Germany: Pabst.

Schmidt, W. C. (1997). World-Wide Web survey research: Benefits, potential problems, and solutions. Behavior Research Methods, Instruments, and Computers, 29, $274-279$.
Schmidt, W. C. (2000). The server-side of psychology Web experiments. In M. H. Birnbaum (Ed.), Psychological experiments on the Internet (pp. 285-310). San Diego, CA: Academic Press.

Smart, R. (1966). Subject selection bias in psychological research. Canadian Psychologist, 7a, 115-121.

Schwarz, S., \& Reips, U.-D. (2001). CGI versus JavaScript: A Web experiment on the reversed hindsight bias. In U.-D. Reips \& M. Bosnjak (Eds.), Dimensions of Internet Science (pp. 75-90). Lengerich, Germany: Pabst Science.

Securityfocus.com. (n.d.). BUGTRAQ Vulnerability Database Statistics. Retrieved April 7, 2002, from http:// www.securityfocus.com

Voracek, M., Stieger, S., \& Gindl, A. (2001). Online replication of Evolutionary Psychology evidence: Sex differences in sexual jealousy in imagined scenarios of mate's sexual versus emotional infidelity. In U.-D. Reips \& M. Bosnjak (Eds.), Dimensions of Internet Science (pp. 91-112). Lengerich, Germany: Pabst Science.

Wenzel, O. (2001). Webdesign, Informationssuche und Flow: Nutzerverhalten auf unterschiedlich strukturierten Websites [Web design, search for information, and flow: User behavior on differently structured Web sites]. Lohmar, Germany: Eul.

\section{Ulf-Dietrich Reips}

Experimental and Developmental Psychology

University of Zürich

Attenhoferstr. 9

CH-8032 Zürich

Switzerland

Tel.: +41 16342930

Fax: +41 16344929

E-mail: ureips@genpsy.unizh.ch 